\title{
Forza Italia zwischen Volks- und Kartell-Partei. Ein Beispiel für Anwendungs-, nicht Theoriedefizite in der Parteienforschung
}

\author{
Eike-Christian Hornig
}

Forza Italia (FI) ist seit einem Dutzend Jahren, trotz des Regierungsverlustes im April 2006, bei Wahlen die erfolgreichste Partei in Italien und damit eine der wichtigsten europäischen Parteien. Dieser anhaltende Erfolg ${ }^{1}$, die Besonderheiten der Organisationsstruktur, die Entstehungsumstände und die Rolle von Silvio Berlusconi als Übervater sind eine fortdauernde Herausforderung für die politikwissenschaftliche Forschung. Obwohl Forza Italia nicht nur in den großen Monographien von Emanuela Poli ${ }^{2}$ und Damian Grasmück ${ }^{3}$ detailliert analysiert worden ist, muss die parteientheoretische Auseinandersetzung mit FI realistischerweise als gescheitert betrachtet werden. Die verbreitete biographische Perspektive über Silvio Berlusconi ${ }^{4}$ und die Überproduktion neuer Begriffe für den Parteityp belegen dies eindrucksvoll: „virtuelle Partei, Unternehmerpartei, Medienpartei, Ein-Mann-Partei, Scheinpartei, Antiparteien-Partei“5, „charismatische Partei“6, „Persönlichkeitspartei“ beziehungsweise „media-mediated-party“7, „Bewegungspartei“8, „Medienkommunikationspartei“9 oder "Polit-Bewegung" 10 . Paolo Alberti bringt dieses Problem der Forschung auf den Punkt: „Genauere Betrachtungen der FI hinterlassen den Eindruck, wir hätten es mit einem neuen Phänomen zu tun, das sich den traditionellen Kategorien politikwissenschaftlicher Analyse entzieht. Diese Einschätzung hat zu einer Hochkonjunktur neuer Definitionen sowie der Schöpfung neuer Parteimodelle geführt, die nicht von einem sparsamen Umgang mit Begriffen dieser Art geprägt sind (...)."11

Diese Modelle und Termini sind dabei im Einzelfall und in der Summe schlüssig und trügerisch zugleich, wie am Beispiel der „charismatischen Partei“ aus dem Jahr 2005 deutlich wird. Sie ist in etwa bedeutungsgleich mit der „Persönlichkeitspartei“ aus dem Jahr

1 Bei den Parlamentswahlen am 9./10. April erzielte FI mit 23,7 Prozent der Stimmen für die Abgeordnetenkammer und 24 Prozent für den Senat das beste Einzelergebnis. Vgl. Stefan Köppl, Machtwechsel um Haaresbreite: Die Parlamentswahlen in Italien vom 9./10. April 2006, in: ZParl, 37. Jg. (2006), H. 4, S. 758.

2 Emanuela Poli, Forza Italia - Strutture, leadership e radicamento territoriale, Bologna 2001.

3 Damian Grasmück, Forza Italia Silvio Berlusconis. Geburt, Entwicklung, Regierungsfähigkeit und Strukturen einer charismatischen Partei, Frankfurt am Main 2005.

4 Wie es Stefan Köppl etwa für die Arbeit von Damian Grasmück festhält. Stefan Köppl, Besprechung von Damian Grasmück: die Forza Italia Silvio Berlusconis, in: PVS, 47. Jg. (2006), S. 132 f.

5 Paolo Alberti, Forza Italia - neuer Wein in alten Schläuchen. Eine klassische catch-all-Partei mit einem neuen Modell politischer Legitimation, in: Josef Schmid / Udo Zolleis (Hrsg.), Zwischen Strategie und Anarchie. Der Erfolg von Parteiorganisationen, Wiesbaden 2005, S. 239.

6 Damian Grasmück, a.a.O.

7 Jörg Seißelberg, Conditions of Success and political Problems of a 'Media-Mediated PersonalityParty': The case of Forza Italia, in: West European Politics, 19. Jg. (1996), S. 715 - 743.

8 Elisabeth Fix, Italiens Parteiensystem im Wandel, Frankfurt am Main / New York 1999.

9 Uwe Jun, Forza Italia - der Prototyp einer Medienkommunikationspartei?, in: Tobias Dürr / Franz Walter (Hrsg.), Solidargemeinschaft und fragmentierte Gesellschaft, Opladen 1999, S. 475 $-492$.

10 Hans-Joachim Fischer, Kommentar, in: Frankfurter Allgemeine Zeitung vom 12. April 2006, S. 1.

11 Paolo Alberti, a.a.O., S. 239. 
1996. Es wird aber trotz der analytischen Atomisierung nahezu einstimmig betont, dass sich FI nach der Organisationsreform von 1997 durch die Öffnung für eine Massenmitgliedschaft wesentlich verändert hat. ${ }^{12}$ Eine Beurteilung anhand der Persönlichkeit von Silvio Berlusconi und / oder seines Medienimperiums erweist sich also aus parteientheoretischer Sicht als wenig hilfreich. Dagegen öffnet die Einbettung einer Analyse in den Kontext der so genannten party-change-Forschung, wie sie Paolo Alberti jüngst vorgenommen hat, Forza Italia für eine breitere anschlussfähigere ${ }^{13}$ Perspektive. Dabei wurde allerdings festgestellt, dass es sich bei FI ausgerechnet um eine klassische catch-all- beziehungsweise Mitglieder-Partei handeln soll, die nur über ein neues Legitimationsmodell verfügt. So qualifizieren besonders Leistungen außerhalb der Politik zu politischen Führungsrollen. Dagegen wird in der Strategie und Organisation von FI kaum etwas grundsätzlich Neues erkannt. Alberti vertritt mit dieser Einschätzung den mit Abstand konservativsten Ansatz, der einen Widerspruch zu den genannten Parteimodellen formuliert: „Die von der FI eingeführten Innovationen hinsichtlich Strategie und Organisation waren sicherlich nicht der Vorbote eines neuen Parteimodells. Vieles ist im Gegenteil auf das alte Modell der catch-all-Party von Otto Kirchheimer zurückzuführen. Dieses wurde hier mit Marketingtechniken versehen, die für traditionelle italienische Parteien äußerst fortschrittlich und eher unüblich sind. Die angewandten Techniken trieben zwar das Potenzial des, catch-all-Modells' auf die Spitze, doch ihre Ergebnisse lassen sich dennoch auf diesen Parteityp zurückführen. Es handelt sich um eine, Catch-all-Partei' hoch drei, wenn man so will, aber immer noch um eine ,Catch-all-Partei'. "14 Die Formulierung einer „catch-all-Partei hoch drei“ macht stutzig, denn die Steigerung der Eigenschaften der dritten Phase spräche eigentlich für eine vierte Phase, wenn Klaus von Beymes Feststellung stimmt, dass sich im vierten Stadium einige Tendenzen verstärkt haben, die in der dritten Phase angelegt worden sind. ${ }^{15}$ Problematisch ist außerdem, dass bei Alberti die stadientypologische Perspektive sehr auf den catchall-Aspekt verengt und es versäumt wird, die Bewertung von FI als Mitglieder-Partei anhand der tatsächlichen Rolle der Mitgliedschaft zu diskutieren.

Der vorliegende Beitrag möchte diese Fehlentwicklungen insofern korrigieren, als er sich zum einen gegen eine Terminologie sui generis ausspricht und stattdessen die Fruchtbarkeit eines stadientypologischen Ansatzes zur Bewertung von Forza Italia (und darüber hinaus) unterstreichen möchte. Demnach erzeugen die idealtypischen Modelle trotz ihrer Unvollkommenheit ein stabiles Kraftfeld, das eine Positionierung realer Parteien und auch eines Sorgenkindes wie FI erlaubt. Somit kann der Bann der terminologischen und konzeptionellen Isolation gelöst und FI generellen komparativen Dimensionen geöffnet werden. Aus dieser Perspektive soll zum anderen die strukturelle Entwicklung von Forza Italia (Öffnung zur Mitgliedschaft 1997) neu bewertet werden. Eine differenzierte Analyse ihrer binnenorganisatorischen Wandlungen vor dem Hintergrund der idealtypischen Aussagen zur catchall- und Kartell-Partei zeigt, dass die These von Alberti, dass es sich bei Forza Italia um „eine

12 Ebenda, S. 243; Elisabeth Fix, a.a.O., S. 220; Damian Grasmück, a.a.O., S. 385; Emanuela Poli, a.a.O., S. 132.

13 So hat Klaus Detterbeck bereits 2002 acht westeuropäische Parteien auf ihre Übereinstimmung mit der Kartell-Parteien-These untersucht. Klaus Detterbeck, Der Wandel politischer Parteien in Westeuropa, Opladen 2002.

14 Paolo Alberti, a.a.O., S. 240.

15 Klaus von Beyme, Parteien im Wandel. Von den Volksparteien zu den professionalisierten Wählerparteien, Wiesbaden 2000, S. 34. 
klassische catch-all-Partei"16 handeln soll, nicht zu halten ist. Vielmehr spricht ihre Entwicklung für eine Annährung an die so genannte Kartell-Partei. Damit wird zugleich eine Gegenposition zu den Versuchungen eines konzeptionellen „anything-goes“ formuliert, wie sie die „catch-all-Partei hoch drei“" andeutet.

\section{Zur Operationalisierung der Parteienwandelforschung}

In verschiedenen idealtypischen Stadienmodellen werden die Wandlungsprozesse der Organisationen, Funktionen und Inhalte politischer Parteien seit dem Ende des 19. Jahrhunderts reflektiert. Nach der Devise „Typenbildung ist Generalisierung auf mittleren Erfahrungswerten "17 zielen sie darauf ab, den durchschnittlichen Entwicklungsstand widerzuspiegeln. So folgt nach der Honoratioren- und der ideologischen Massenpartei in den 1950ern die Volkspartei als dominierender Typus. ${ }^{18}$ Während die ersten Phasen kaum mehr Anlass zur Diskussion geben, steht seit 1995 die These der Kartell-Partei ${ }^{19}$ von Richard S. Katz und Peter Mair als vierte Phase im Raum. Trotz zahlreicher Kritik ${ }^{20}$ ist diese als Kristallisationspunkt und Stimulus zu einem Klassiker der jüngeren Parteienforschung geworden. ${ }^{21}$

Eine Operationalisierung von Parteienwandel muss viele methodische und inhaltliche Einschränkungen hinnehmen, die dem Wunsch nach möglichst präzisen Bemessungen Restriktionen auferlegen, denn die Stadientypologie ist weder ein einheitliches Konzept noch unumstritten. Erstens ist grundsätzlich zu beachten, dass es sich bei der Parteientypologie um ein (nicht-normatives) theoretisches Phänomen handelt, das als Idealtypus im Sinne Max Webers durch die rein gedankliche Steigerung bestimmter Elemente der Wirklichkeit gewonnen wird. ${ }^{22}$ Zweitens sind die Entwicklungsphasen nicht ausschließlich zu verstehen, sondern es handelt sich um den jeweils dominanten Typus. Klaus von Beyme weist darauf hin, dass keine der vier Phasen je eine ganze Epoche und alle wichtigen Länder mit ihren Parteien umfasst hat. ${ }^{23}$ Drittens entsprechen den Mustern der Stadientypologie in der

16 Paolo Alberti, a.a.O., S. 238.

17 Klaus von Beyme, a.a.O., S. 28.

18 Otto Kirchheimer, The Transformation of the Western European Party System, in: Joseph LaPalombara / Myron Weiner (Hrsg.), Political Parties and Political Development, Princeton 1966, S. $177-200$.

19 Richard S. Katz / Peter Mair, Changing Models of Party Organization and Party Democracy. The Emergence of the cartel party, in: Party politics, 1. Jg. (1995), S. 5-28.

20 Zur Rezeption der Kartell-Parteien-These in der deutschen Politikwissenschaft von 1995 bis 2001: Ludger Helms, Die Kartell-Parteien-These und ihre Kritiker, in: PVS, 42. Jg. (2001), S. 698 -708 .

21 Ebenda, S. 707.

22 Die Einwände gegen die Inanspruchnahme des Weberschen Idealtypus für die Stadientypologie zielen auf die Unterschiedlichkeit der damit beschriebenen Phänomene und kritisieren zwischen den Zeilen eine zu leichtfertige Nutzung. So weist Beyme stellvertretend darauf hin, dass Max Weber mit dem Idealtypus eigentlich Abstraktionen wie Stadt oder Kapitalismus im Sinne hatte: „Von einer ,Steigerung der Merkmale', die Weber mit dem Idealtyp verband, kann keine Rede sein. Für so wenig komplexe Begriffe wie die Typen der Parteien reicht der deskriptive Realtyp aus." Klaus von Beyme, a.a.O., S. 27.

23 Ebenda, S. 208. 


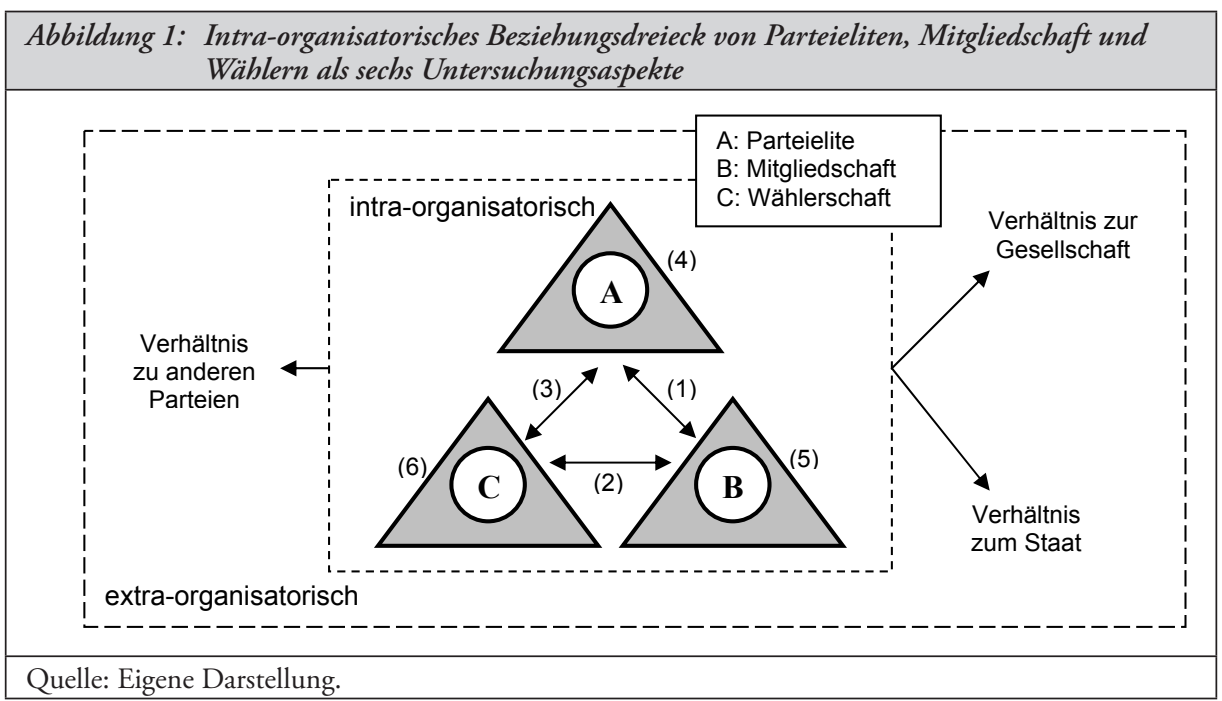

Mehrzahl der Realtypen nur große, europäische Parteien. ${ }^{24}$ Viertens kennzeichnen Überlappungen beziehungsweise fließende Übergänge die Phasen. Da klare Einschnitte fehlen, sollte Datierungsversuchen skeptisch begegnet werden. Trotz der Unvollkommenheit der Stadientypologie als "patch-work-Theorie“ ist sie bislang die einzige brauchbare Antwort auf die Frage, ,warum ein bestimmter Organisationstyp in einer Epoche überwiegt" ${ }^{\text {"25. }}$.

Die wesentliche Funktion politischer Parteien in pluralistischen Demokratien ist die Verbindung der Gesellschaft mit den staatlichen Institutionen. Diese unterliegt einer Entwicklungsdynamik, da gesellschaftliche Modernisierungsschübe die Anforderungen verändern. Hierzu gehören die Erweiterung des Wahlrechts, der Wandel der „cleavage“-Struktur, die fortschreitende Entideologisierung, die Einführung der staatlichen Parteienfinanzierung oder der so genannte Medienwandel. ${ }^{26}$ Diese ,intervenierenden Variablen “27, wie Beyme sie nennt, verändern die Position der Parteien zwischen Staat und Gesellschaft, aber auch die innere Rollenverteilung zwischen der Parteielite, der Parteimitgliedschaft und der Wählerschaft, so dass sich der Parteienwandel analytisch auf einer extra- und einer intra-organisatorischen Ebene manifestiert. Der Blick ins Innere der Parteien ist dabei für eine brauchbare Stadientypologie unabdingbar.

Die in Abbildung 1 dargestellten sechs Untersuchungsaspekte sollen der Analyse der organisatorischen Veränderungen von Forza Italia zu Grunde gelegt werden: erstens das Verhältnis zwischen Parteielite und Mitgliedern, zweitens jenes zwischen Mitgliedern und

24 Frank Decker, Parteien und Parteiensysteme im Wandel, in: ZParl, 30 Jg. (1999), H. 2, S. 355.

25 Klaus von Beyme, a.a.O., S. 27.

26 Als Konkretisierung des Begriffes Medienwandel führt Barbara Pfetsch die Einführung neuer Medientechnologien sowie die Deregulierung der Kommunikationsinfrastruktur und des Rundfunksystems an. Daraus geht die Expansion der Medienangebote, ihre Kommerzialisierung und Beschleunigung hervor. Barbara Pfetsch, Regieren unter den Bedingungen medialer Allgegenwart, in: Ulrich Sarcinelli (Hrsg.), Politikvermittlung und Demokratie in der Mediengesellschaft, Bonn 1998, S. 233 - 252, S. 243.

27 Klaus von Beyme, a.a.O., S. 28. 
Wählern und drittens zwischen Parteielite und Wählern. Da die drei Teileinheiten im Verlauf der Entwicklung nicht nur ihre Position innerhalb des Dreiecks verändern, sondern auch ihre Charakteristika einem Wandel unterworfen sind, können drei weitere Indikatoren hinzugezählt werden: viertens die Veränderungen der Parteielite, fünftens die Veränderung der Mitgliedschaft und schließlich die Veränderungen in der Wählerschaft. ${ }^{28}$

\section{Prozesse des stadientypologischen Wandels}

Am Anfang der parteientypologischen Entwicklung standen die Honoratioren-, Elite- oder Kaderparteien der zweiten Hälfte des 19. Jahrhunderts, die durch die Universalisierung des Wahlrechts ihr Ende fanden. ${ }^{29}$ Sie hatten kaum Ähnlichkeit mit den heutigen Parteien. Weder verfügten sie über eine nennenswerte (formalisierte) Mitgliedschaft, noch über verfestigte organisatorische Strukturen. Vielmehr handelte es sich um einen kleinen, losen (Männer-)Verbund von Mitgliedern der (Besitz-)Oberschicht, die einzelne ihrer Exponenten in staatliche Ämter entsandten. Als Qualifikation reichte die Mitgliedschaft zur privilegierten Klasse aus. Mit ihrem Engagement betrieben sie im Wesentlichen Privilegiensicherung. ${ }^{30}$ Dabei war keine Rückanbindung der prinzipiell unabhängigen Abgeordneten an die Partei(-mitgliedschaft) notwendig, da es diese im heutigen Sinne nicht gab. Aufgrund des beschränkten Wahlrechts spielte auch der Wahlkampf kaum eine Rolle. Es gab zwar vor Ort einige dominierende Honoratioren, aber keine organisierte und gewählte Elite, die die Geschicke der Organisation steuerte, da es auch kaum organisatorische Strukturen gab. Es lässt sich also kaum ein relevantes Kräfteverhältnis der Teileinheiten ausmachen, da diese nicht eindeutig voneinander zu trennen sind. ${ }^{31}$

Das zweite Entwicklungsstadium kennzeichnet der Übergang zur Massenmitgliedschaft. Hierbei handelte es sich insbesondere um Arbeiter, die das gesellschaftliche Bild europäischer Industrienationen zum Ende des 19. und zu Beginn des 20. Jahrhunderts prägten. Die Massenparteien, die ihren Höhepunkt in der Zwischenkriegszeit erreichten ${ }^{32}$, basierten auf scharfen Klassenkonflikten und setzten den vorherrschenden Zuständen ihre alternativen ganzheitlichen Gesellschaftskonzepte entgegen. Zu deren Umsetzung entwickelten sie eine breite und hierarchische Organisationsstruktur, in der die Massenmitgliedschaft zu der entscheidenden Instanz wurde, die die Führung von unten kontrollierte. ${ }^{33}$ Mandatsträger der ideologisierten Massenparteien handelten als Delegierte streng im Sinne ihrer Mutter-

28 Dieser Ansatz baut auf einen breiten intra-organisatorischen Begriff, während beispielsweise Klaus Detterbeck bei seiner Untersuchung acht ausgewählter westeuropäischer Parteien anhand der Kartell-Parteien-Theorie die Involvierung der Mitglieder vom organisatorischen Wandel trennt und sich auf vier Vergleichspunkte beschränkt: (1) die Kontrolle über die Führungsgremien, (2) die Kandidatenselektion, (3) die Kontrolle über den innerparteilichen Entscheidungsprozess, (4) die Kontrolle über die Organisation des Wahlkampfes. Klaus Detterbeck, a.a.O.

29 Es gab zwar vorher bereits Massenparteien, diese waren von der Führung des Systems in der Regel aber ausgeschlossen. Vgl. Klaus von Beyme, a.a.O., S. 28. Dieser Einschnitt stellt das einzige Beispiel für eine verhältnismäßig präzise Terminierung des Stadienwandels dar.

30 Ebenda, S. 41.

31 Daher kann diese Phase auch nicht in der Abbildung 2 dargestellt werden.

32 Vgl. Klaus Detterbeck, a.a.O., S. 25.

33 Vgl. Klaus von Beyme, a.a.O., S. 41. 
organisationen. Allerdings zeigten sich auch erste Anzeichen einer Professionalisierung im Sinne eines Berufspolitikertums. ${ }^{34}$ Der Wahlkampf gewann in der Phase der Massenparteien an Bedeutung, wobei er weniger der Gewinnung neuer Wähler als der Mobilisierung der Stammwähler aus den zugehörigen gesellschaftlichen Subkulturen diente. Er wurde materialintensiv geführt und beanspruchte ein breites (auch finanzielles) Engagement der Mitgliedschaft und erhöhte Koordinierungsanforderungen, denen die Parteielite durch den Ausbau parteiinterner Kommunikationsnetze (Parteizeitungen) begegnete.

Für das Beziehungsdreieck von Parteielite, Mitgliedschaft und Wählerschaft war in der Ära der ideologisierten Massenpartei charakteristisch, dass die drei Teileinheiten klare Konturen entwickelten. Dabei lässt sich für die Parteielite ein erster Professionalisierungsschub festhalten, der sowohl die Wege der Kommunikation als auch den Status der Funktionäre betraf. Geprägt wurde diese Phase allerdings durch die Parteimitgliedschaft, die als maßgebliche Instanz das gesamte Dreieck dominierte. Die Wählerschaft war eine Stammwählerschaft, die sich aus den ideologisch kompatiblen Subkulturen erschloss. Dementsprechend standen im Kräftefeld die Mitglieder zwischen der Parteielite und den Wählern, das heißt die Distanz zwischen den letzten beiden war die weiteste im Dreieck, während jene zwischen der Mitgliedschaft und der Elite die kürzeste war.

Während die Ausweitung des allgemeinen Wahlrechts im Übergang von der Honoratiorenpartei zur ideologisierten Massenpartei einen recht deutlichen Einschnitt darstellt, gestaltet sich der Übergang zur Phase der Volksparteien in seiner zeitlichen Determinierung schwieriger. Das Ende des Zweiten Weltkrieges bildet zwar eine deutliche Zäsur, ist aber nicht gleichbedeutend mit dem Übergang zur Volkspartei ${ }^{35}$, die als dritte stadientypologische Phase auf Otto Kirchheimer zurückgeht. ${ }^{36}$

In der Folge gesellschaftlicher Modernisierungsschübe und steigender ökonomischer Saturierung kam es zur Entschärfung der Klassenkonflikte, zur Entideologisierung der Wählerschaft und ihrer steigenden politischen „Verfügbarkeit“. Die sich analog dazu etablierende Volks- oder catch-all-Partei spiegelte diese gesellschaftlichen Entwicklungen wider, indem sie die Verfügbarkeit aufgriff und sich als Strategie zu Grunde legte. So kam es zu einer Annäherung zwischen der Parteielite und der Wählerschaft, da sich erstere wesentlich mehr als zuvor an letzterer orientierte, wie der Wahlkampf zeigte, der mit einem entideologisierten Programm ein Stimmenmaximierungskonzept verfolgte und für den die Volkspartei den Großteil ihres Potenzials aufwendete. Aufgrund der zunehmenden Dominanz der Massenmedien (Fernsehen) ab den 1950er Jahren erhöhten sich die kommunikativen Ko-

34 So schreibt Hans H. Klein in diesem Zusammenhang: „Zunehmend fanden die so genannten Privatbeamten den Weg ins Parlament: Partei- und Verbands-, und insbesondere Gewerkschaftsfunktionäre, deren politisches Engagement von den Gruppen, deren Interessen sie wahrnahmen, finanziell entgolten wurde." Hans H. Klein, Mandat und Beruf, in: Frankfurter Allgemeine Zeitung vom 8. August 2006, S. 7.

35 Bernd Hoffmann setzt den Einschnitt mit der Nachkriegszeit (Bernd Hoffmann, Annährung an die Volkspartei. Eine typologische und parteiensoziologische Studie, Wiesbaden 2004, S. 42), während Klaus von Beyme eher die Mitte der 1950er Jahre in den Vordergrund stellt. Neben unterschwelligen gesellschaftlichen Veränderungen, die sich in der Kunst, Architektur und Literatur manifestierten, fand demnach auch ein Modernisierungsschub der Denk- und Verhaltensweisen statt. Klaus von Beyme, a.a.O., S. 29. Karsten Rudolph spricht von den 1960er Jahren sogar als dem Jahrzehnt der Volksparteien. Karsten Rudolph, Die sechziger Jahre: Das Jahrzehnt der Volksparteien?, in: ZParl, 30. Jg. (1999), H. 2, S. 362 - 376.

36 Otto Kirchheimer, a.a.O. 
ordinationsanforderungen. Was in einem überschaubaren Rahmen begann, führte zu einer „Unterordnung des Wahlkampfes unter die massenmedialen Produktionsgesetze“37. Die Parteien mussten immer mehr ihr Know-how vertiefen und gleichzeitig externe Experten wie Werbeagenturen und Wissenschaftler zu Rate ziehen. Die Professionalisierung der Parteielite, insbesondere durch das Wachstum der Mitarbeiterstäbe der Abgeordneten, erreichte eine neue Stufe.

In demselben Maße, wie die Parteielite an die Wählerschaft heranrückte, entfernte sie sich von der Mitgliedschaft. Diese verlor an organisationsinterner Bedeutung und näherte sich wiederum der Wählerschaft an. Der Bedeutungsverlust rührte aus den sich wandelnden Anforderungen des Wahlkampfes, bei dem das einzelne Parteimitglied weniger wichtig war als etwa die Strategen in den Parteizentralen. Außerdem trat die Mitgliedschaft ab Mitte der 1960er Jahre durch die einsetzende staatliche Parteienfinanzierung, die die Parteien - im Sinne ihrer Erhebung in den Verfassungsrang - als Träger des demokratischen Prozesses stärken sollte, auch als finanzielle Ressource in den Hintergrund. ${ }^{38}$ Die Einnahmenstruktur wurde mit Mitgliedsbeiträgen, Spenden und staatlichen Zuwendungen zu einem Drei-Säulen-Modell. ${ }^{39}$ Hinzu kam die Professionalisierung der Parteieliten, die in Verbindung mit der Kopplung von Parteiämtern und öffentlichen Mandaten zu einem verstärkten Machtgefälle führte. Diese Entwicklungen wurden allerdings in einigen Ländern durch innerparteiliche Demokratisierung kontrastiert. ${ }^{40}$ Auch waren die Mitglieder als Repräsentanten der Partei in der Gesellschaft als Quelle demokratischer Legitimation und über die Hebel der organisationsinternen Demokratie nach wie vor in einer mächtigen Vetorolle. Dennoch ist die Volkspartei zunehmend von Mitgliederschwund betroffen, was immer wieder die Diskussion um die Zukunft der Mitgliedschaft in politischen Parteien anfacht. ${ }^{41}$

Angelo Panebianco konstatierte im Jahr 1988 mit einer Aktualisierung des Volksparteienmodells zur so genannten electoral-professional party eine abermalige Professionalisierung der Parteieliten und -organisationen. ${ }^{42}$ „Diese unterschied sich im Wesentlichen durch die (...) Ersetzung der alten Parteibürokraten durch akademisch ausgebildete Fachexperten von Kirchheimers catch-all-party. " 43 Auch wenn betont wird, dass sie ansonsten nach wie vor der Volkspartei entspricht ${ }^{44}$, ist sie Indiz für die Weiterentwicklung der Stadientypologie,

37 Bernd Hoffmann, a.a.O., S. 111.

38 Nach der Bundesrepublik 1959 folgten 1965 Schweden, 1967 Finnland, 1970 Norwegen, 1974 Italien, 1975 Österreich, 1977 Spanien und 1986 Dänemark und Frankreich, vgl. Klaus von Beyme, a.a.O., S. 134.

39 Hoffmann hält aber fest, dass es bezüglich der Anteile kein Maß gibt, das eine klare Trennung zwischen den Phasen erlauben würde. Bernd Hoffmann, a.a.O., S. 111.

40 Diese sieht Klaus von Beyme als eine Folge der Protestbewegung der 1960er Jahre. Doch vollzog sie sich nicht linear und wurde zum Teil wieder zurückgebaut, wie das Beispiel der Kanzlerkandidatenauslese in der SPD zeigt. So wurde Rudolf Scharping 1994 durch ein Mitgliederreferendum, Gerhard Schröder 1998 dagegen durch eine Parteitagsentscheidung gekürt. Klaus von Beyme, a.a.O., S. 33. Klaus Detterbeck weist vor diesem Hintergrund darauf hin, dass die deutschen Parteien davor zurückschrecken würden, mehr Partizipation der Mitglieder in der Parteiarbeit zu ermöglichen. Klaus Detterbeck, a.a.O., S. 73.

41 Vgl. hierzu Elmar Wiesendahl, Mitgliederparteien am Ende? Eine Kritik der Niedergangsdiskussion, Wiesbaden 2006.

42 Angelo Panebianco, Political Parties: organization and power, Cambridge 1988.

43 Klaus Detterbeck, a.a.O., S. 28.

44 Angelo Panebianco, a.a.O., S. 311. 
die 1995 mit der Kartell-Parteien-These ${ }^{45}$ als vierter Stufe von Katz und Mair fortgeschrieben wurde.

Stand bei den ersten drei Phasen die Beziehung zwischen Parteien und Gesellschaft im Vordergrund, „rückt das Kartellparteien-Modell von Richard S. Katz und Peter Mair die Bindung politischer Parteien an den Staat in den Mittelpunkt der Aufmerksamkeit "46. Sein Kern ist die These, dass sich die gesellschaftliche Verwurzelung der Parteien erheblich gelöst habe und diese sich stattdessen zunehmend dem Staat als Ressourcenquelle zuwendeten. Daher seien sie in der vierten Phase nicht mehr Teil der Gesellschaft wie in der ersten und zweiten Phase, nicht mehr Vermittler zwischen Gesellschaft und Staat wie in der dritten Phase, sondern selbst Teil des Staates. ${ }^{47}$ Die Implikationen dieser These sollen für das Beziehungsdreieck von Parteielite, Mitgliedschaft und Wählern als eines von vier Elementen der Kartell-Parteien-These ${ }^{48}$ für die Untersuchung von FI aufgegriffen werden.

Die Globalisierung der Wirtschaft und Kommunikation, das Abschwächen der klassischen Konfliktlinien, die Entstehung von Dienstleistungsgesellschaften und die Individualisierung der Lebensentwürfe hat breite Schichten entideologisierter mittelständischer Angestellter mit schwindender Parteiidentifikation geschaffen, während die klassischen Milieus der Parteien immer kleiner werden. ${ }^{49}$ Kennzeichen der Veränderung für den Bereich der Wählerschaft ist eine gegenüber der Volkspartei nochmals gewachsene Verfügbarkeit, die sich in einer steigenden Volatilität manifestiert. ${ }^{50}$ Auch die Parteielite hat sich verändert und hat eine neue Stufe der Professionalität erreicht, die in Zeiten massenmedialer Werbeschlachten und bürokratischer Parteiapparate zwar kein originäres Charakteristikum der vierten Phase ist, sich aber in ihrer Dimension am Ende des 20. Jahrhunderts wesentlich von der Volksparteienphase unterscheidet. Diese wahlkampfzentrierte Parteiarbeit ist sehr kapitalintensiv, da aber der Geldfluss der Mitgliedschaft geringer wird, haben die Parteien mit der staatlichen Finanzierung eine neue zuverlässige Quelle gefunden beziehungsweise

45 Der Begriff der Kartell-Partei ist aufgrund seines Verwechlungspotentials kritisiert worden. Da auch die Kader- und Honoratiorenparteien im frühen 20. Jahrhundert so bezeichnet wurden, zieht Beyme, in Anlehnung an Panebianco, den Begriff der professionalisierten Wählerpartei vor. Andere Begriffe wie Medienpartei, Minimalpartei oder Rahmenpartei haben sich aufgrund ihrer Eindimensionalität oder Widersprüchlichkeit ebenfalls als wenig hilfreich erwiesen. So ist etwa der Begriff der Minimalpartei irreleitend, da die Parteien im Hinblick auf ihre Durchdringung von Politik und Gesellschaft eher einen maximalen Charakter aufweisen. Klaus von Beyme, a.a.O., S. 36.

46 Thomas Poguntke, Zur empirischen Evidenz der Kartellparteien-These, in: ZParl, 33. Jg. (2002), H. 4, S. $790-806$, S. 790 f.

47 Richard S. Katz / Peter Mair, a.a.O, S. 18.

48 Zweitens geht es um das Verhältnis zwischen Gesellschaft, Staat und Parteien. Die Verbindungen der Repräsentanten dieses Parteientyps untereinander markieren den dritten Punkt, während das Verhältnis der Kartellparteien zu anderen Parteien und die Beeinflussung von deren Erfolgsaussichten die vierte Dimension darstellen. Vgl. Ludger Helms, a.a.O., S. 699.

49 So ist die quantitative Marginalisierung der einstigen Trägerschichten in den Parteimitgliedschaften der Bundesrepublik mehr das Produkt sinkender Gruppengröße als nachlassender Prägekraft der Milieus, da die traditionellen Trägerschichten sich weiterhin in ,ihren' Parteien organisieren. Heiko Biehl, Wie viel Bodenhaftung haben die Parteien? Zum Zusammenhang von Parteimitgliedschaft und Herkunftsmilieu, in: ZParl, 37. Jg. (2006), H. 2, S. 277 - 292, S. 291.

50 Im Durchschnitt von zunächst 15, dann 18 westeuropäischen Ländern wächst die Wählerfluktuation in Parteiensystemen zwischen 1950 und 1999 (Nettogewinne) von anfangs 7,5 auf 10,25 Prozent zwischen 1991 und 1999 an. Vgl. Klaus von Beyme, a.a.O., S. 59 f. 
geschaffen: „Parties have therefore been obliged to look elsewhere for their resources and in this case their role as governors and law-makers made it easy for them to turn to the state. "51 Die staatliche Finanzierung ist nach ihrer flächendeckenden Etablierung in der Phase der Kartell-Parteien zu ihrer wesentlichen Einnahmequelle geworden ${ }^{52}$, auch wenn Beyme einwendet, dass sich seit Mitte der 1970er keine lineare Entwicklung zu mehr Staatsfinanzierung der Parteien feststellen lässt. ${ }^{53}$

Die zunehmende fachliche und finanzielle Unabhängigkeit der Parteiführung steht in einem reziproken Verhältnis zum Funktionswandel der Mitglieder: Sie verlieren als zentraler Bezugspunkt der Parteieliten immer mehr an Bedeutung. Die staatliche Parteienfinanzierung trägt zu einer Abschottung der Parteiführung von den Mitgliedern bei, auch wenn sie so nicht intendiert war. ${ }^{54}$ Immer weniger Parteimitglieder sehen noch die Notwendigkeit, sich finanziell und aktiv an der Parteiarbeit zu beteiligen. Tun sie es doch, sind es immer seltener inhaltliche (früher ideologische), sondern meistens Karriere-Motive. Für Katz und Mair unterliegt die Mitgliedschaft mit der „atomistic conception of party member-ship “55 auch einer neuen Logik. Die Mitglieder haben in den Kartell-Parteien vielleicht mehr Rechte als in der Volkspartei - dennoch nähern sie sich dem Status der Wähler immer mehr an. Mechanismen parteiinterner Demokratie, zum Beispiel durch Briefwahl, lösen zunehmend das Delegiertensystem auf Parteikongressen ab. Finden doch derartige Veranstaltungen statt, sind alle Unterstützer aufgerufen; die formale Mitgliedschaft tritt in den Hintergrund. Die direkte, massenmediale und mitgliedschaftsunabhängige Ansprache durch die Parteiführung und die direkten Beteiligungsmodi schwächen den organisatorischen Mittelbau. Gleichzeitig wird die Position der Parteiführung gefestigt, da „an atomized membership is less likely to provide the basis for the mobilization of challenges" ${ }^{56}$. Beyme unterstützt diese Argumentation, indem er feststellt, dass schon im Zeitalter der Volksparteien die innerparteiliche Demokratie selten verwirklicht wurde und sie in der vierten Phase eher im Abnehmen begriffen ist. ${ }^{57}$ Josef Schmid und Udo Zolleis weisen aber darauf hin, dass die Mitgliedschaft aufgrund ihrer Vetorolle, der Notwendigkeit der demokratischen Legitimation der Parteien und der Wählermobilisierung trotzdem eine zentrale Größe bleibt. ${ }^{58}$

Wie aus Abbildung $2^{59}$ ersichtlich, bewegen sich die Teileinheiten Parteielite, Mitgliedschaft und Wählerschaft zwischen zwei Polen, deren Anordnung in der Grafik frei gewählt ist. Der Ausgangspunkt liegt auf der rechten Seite in den Kerneigenschaften der Teileinheiten zu Beginn der Entwicklung. Die Parteielite wird als unkoordiniert, die Parteimitgliedschaft als dominierend und die Wählerschaft als Stammwählerschaft charakterisiert.

51 Richard S. Katz / Peter Mair, a.a.O., S. 15.

52 Da diese ex post-Finanzierung an den Wahlerfolg oder an die parlamentarische Repräsentation gebunden ist, sichert sie den Status quo der bereits erfolgreichen Parteien ab. Diese stärken sich so für die Auseinandersetzung mit neuen Parteien und bilden in diesem Sinne ein Kartell.

53 Klaus von Beyme, a.a.O., S. 141.

54 Vgl. ebenda, S. 39, S. 142.

55 Richard S. Katz / Peter Mair, a.a.O., S. 21.

56 Ebenda, S. 21.

57 Vgl. Klaus von Beyme, a.a.O., S. 30.

58 Vgl. Josef Schmid / Udo Zolleis, Zwischen Strategie und Anarchie. Der Erfolg von Parteiorganisationen, in: dies. (Hrsg.), a.a.O., S. 9 - 21, S. 18.

59 Die Darstellung basiert auf einer qualitativen Einschätzung, keiner empirisch-mathematischen Grundlage und ist daher rein heuristischer Natur. 


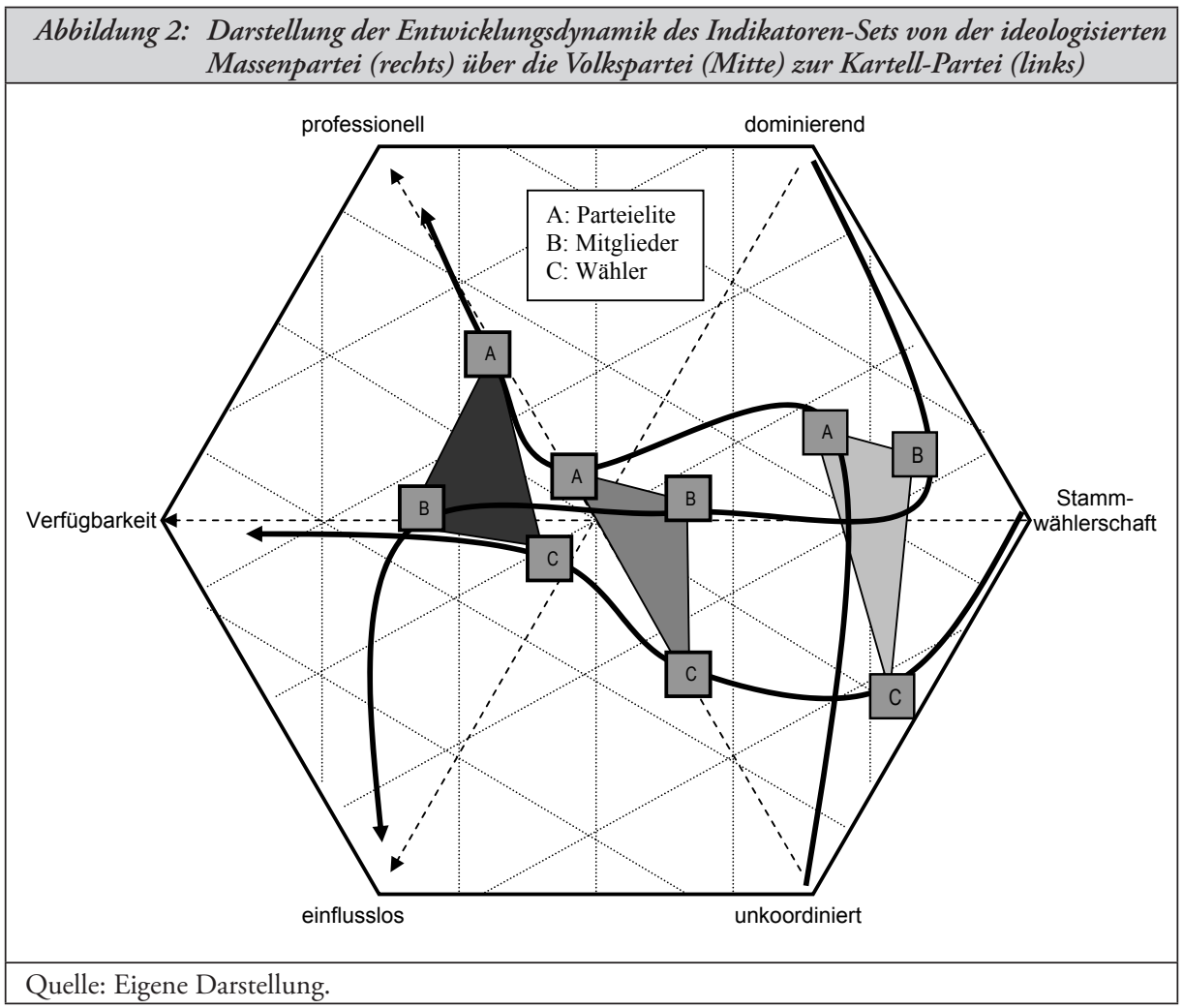

\section{Forza Italia in der Analyse}

\subsection{Gründungsphase ${ }^{60}$}

Die Entstehung von Forza Italia muss vor dem Hintergrund der Veränderungen in der politischen Landschaft Italiens zu Beginn der 1990er Jahre gesehen werden. Unter dem Begriff Tangentopoli ${ }^{61}$ breitete sich eine Prozesswelle über das Land aus, die die politischen Eliten zu Fall brachte. Erste offenkundige Reaktionen der Wähler manifestierten sich in der Parlamentswahl 1992, als den beteiligten Parteien zunehmend das Vertrauen entzogen wurde, wobei besonders die Democrazia Cristiana (DC) als wichtigste Partei die größte Niederlage einstecken musste. ${ }^{62}$ Erreichte 1992 die Volatilität noch 14,4 Prozent, stellten die Wahlen von 1994 den Höhepunkt der Entwicklung dar. Nicht nur lag der Volatilitätswert

60 Da die strukturelle Entwicklung von Forza Italia mit der Gründung 1994 und der großen organisatorischen Reform 1997 zwei Fixpunkte kennt, wird hier eine entsprechende Einteilung in zwei Phasen vorgenommen.

61 Im Jahr 1992 wurden von Mailänder Staatsanwälten die Verstrickungen der Parteien in ein Netz schwarzer Kassen offen gelegt. Betroffen waren fast alle Parteien, besonders aber die Sozialisten (PSI). Vgl. Vittorio Bufacchi / Simon Burgess, L'Italia contesa, Rom 2002, S. 92.

62 Giorgio Galli, I partiti politici italiani, Mailand 2001, S. 384. 
(Abgeordnetenkammer) bei 36,7 Prozent ${ }^{63}$, sondern es wurden auch 71,3 Prozent der Abgeordneten ausgetauscht. ${ }^{64}$ Die Krise bescherte Forza Italia den Wahlsieg. ${ }^{65}$ Sie stieß in die Lücke, die nach der Auflösung der DC zu Beginn des Jahres 1994 entstanden war.

Bei der Gründung und dem zügigen Aufbau der Partei griff Silvio Berlusconi auf die Ressourcen seines Firmenimperiums Fininvest zurück. ${ }^{66}$ Dessen Topmanager wurden zum Grundgerüst der Organisationsstruktur von Forza Italia. Es handelte sich zum größten Teil um Marketing- und Wirtschaftsexperten, die die nicht vorhandenen Parteifunktionäre und Mitglieder ersetzten und das politische Projekt von Berlusconi in der Gesellschaft verankern sollten. ${ }^{67}$ In dieser Phase war Forza Italia eine Kopie der Fininvest, deren Organisationsmerkmale Emanuela Poli benennt ${ }^{68}$ : (1) eine möglichst geringe Anzahl von Mitarbeitern; (2) die mittlere Managementstufe sollte in geringem Umfang vorhanden sein; (3) insgesamt sollte zur Gewährleistung großer vertikaler und horizontaler Mobilität der Mitarbeiter nur eine reduzierte Hierarchie bestehen. Dadurch waren Fininvest und Forza Italia das genaue Gegenteil einer bürokratischen Großorganisation. Da nicht nur die Formen kopiert, sondern auch viele Angestellte der Fininvest die ersten Aktivisten von Forza Italia wurden, konnte der Führungsstil von Berlusconi direkt übertragen werden. Trotz der flachen Hierarchie der Fininvest spricht Elisabeth Fix von einem zentralistischen top-down-Modell. ${ }^{69}$

Das dritte wesentliche Merkmal der Gründung von Forza Italia ergibt sich direkt aus den beiden vorherigen: die extrem hohe Professionalität beim Aufbau der Organisation und beim Wahlkampf. Beides ging nach denselben Regeln vor wie bei der Einführung eines neuen Produktes: Marktuntersuchungen und Meinungsbefragungen spielten die entscheidende Rolle. Die Auswahl der Kandidaten für die Parlamentswahl 1994 erfolgte durch Publitalia, das mit seinem Topmanagement aus über 60 Vertretern in das Projekt Forza Italia eingebunden war. ${ }^{70}$ Dass die nach Marketinggesichtspunkten ausgewählten Kandidaten zu 91,4 Prozent Parlamentsneulinge waren ${ }^{71}$, spielte kaum eine Rolle. Da sie auf die Organisations- und Wahlkampfführung keinen Einfluss hatten, waren sie Statisten. Viel wichtiger war die kurzerhand gegründete Marktforschungsfirma Diakron. Ab Oktober 1993 waren über 150 ihrer Mitarbeiter mit Umfragen beschäftigt, um Berlusconi und seinen Planern einen Eindruck von der Wirkung ihrer Kampagnen, der Popularität und themenspezifischen Glaubwürdigkeit von Berlusconi zu geben.

Die Mitgliedschaft spielte in der Konstituierungsphase keine Rolle. Die einzige Form der Beteiligung bestand in den Clubs Forza Italia, die ab dem 25. November 1993 in ganz

63 Alessandro Chiaramonte, Il voto proporzionale: Versa la nazionalizzazione della competizione, in: Roberto D’Alimonte / Stefano Bartolini (Hrsg.), Maggioritario finalmente?, Bologna 2002, S. 165 - 198, S. 185.

64 Hans-Jörg Keller, Der schwierige Weg zur 2. Republik, in: Winand Gellner (Hrsg.), Umbruch und Wandel in westeuropäischen Parteiensystemen, Frankfurt am Main 1995, S. 63 f.

65 Paolo Natale, Una fedeltà leggera: I movimenti di voto nella seconda Repubblica, in: Roberto D'Alimonte / Stefano Bartolini (Hrsg.), a.a.O., S. 283 - 318, S. 299.

66 Forza Italia wurde offiziell am 18. Januar 1994 gegründet. Allerdings begann die Planung schon im Juni 1993. Vgl. Emanuela Poli, a.a.O., S. 43.

67 Vgl. Elisabeth Fix, a.a.O., S. $210 \mathrm{ff}$.

68 Vgl. Emanuela Poli, a.a.O., S. 39.

69 Vgl. Elisabeth Fix, a.a.O., S. 211.

70 Vgl. Emanuela Poli, a.a.O., S. 54.

71 Vgl. Paolo Alberti, a.a.O., S. 248. 
Italien unter der Leitung der Associazione Nazionale dei Club Forza Italia, kurz ANFI, gegründet wurden. Jeder, der die Spielregeln von ANFI befolgte, konnte einen Club gründen und sein Präsident werden. ${ }^{72}$ Entscheidend ist, dass es für die Club-Mitglieder keine Rechte organisationsinterner Demokratie gab, sondern alles im Sinne des top-down-Modells vorgegeben wurde. Die Mitglieder in den Clubs wurden von der Organisationselite einzig in ihrer Mobilisierungsfunktion als wichtige Ressource betrachtet. Sie fungierten als eine Art Vitrine, in der das Produkt Forza Italia, das die meisten bislang nur aus dem Fernsehen kannten, zu einer realen Angelegenheit und sichtbarem Beweis für die wachsende Unterstützung Berlusconis wurde. Außerdem sollten die Kandidaten von Forza Italia (die die Clubs aber nicht auswählen konnten) in den Clubs durch Debatten, Begegnungen und Feste unterstützt und eine gemeinsame politische Identität entwickelt werden. ${ }^{73}$

Zusammenfassend kann gesagt werden, dass Berlusconis Organisation in diesem Zeitraum nach dem Maßstab des parteientypologischen Vergleichs der Abbildung 2 ein echter Extremfall war. Bei der Hälfte der sechs Vergleichspunkte wurden maximale Positionen erreicht. Die Parteielite war so professionell wie eine der größten Firmen des Landes, die Mitgliedschaft spielte organisationsintern absolut keine Rolle, und die Distanz zwischen ihr und der Parteielite erreichte ein Maximum.

\subsection{Konsolidierungsphase}

Schon einige Monate nach dem Wahlerfolg von 1994 entstand Kritik an der „classe dirigente“. Die Hauptpunkte einiger neuer Parlamentarier und Club-Präsidenten waren fehlende innere Demokratie, die dominante Rolle der Fininvest-Mitarbeiter innerhalb von FI sowie organisatorische und strukturelle Schwächen. Auch innerhalb der Fraktion wurde mangelnde Demokratie kritisiert, da sämtliche Führungspositionen per Akklamation durch Berlusconi vergeben wurden und Wahlen nicht stattfanden. Die Reaktion bestand in kleineren Veränderungen an der Oberfläche der Organisationsstrukturen von FI. ${ }^{74}$ Das zwischenzeitlich ins Auge gefasste System der US-amerikanischen Vorwahlen wurde schnell wieder fallen gelassen. Erst die Wahlniederlage 1996 erzwang, dass über ernsthafte Reformen nachgedacht wurde. Berlusconi verkündete, dass FI offener, demokratischer und in der Gesellschaft verwurzelter sein werde. Allerdings sollte die schmale Organisationsstruktur und das

72 Zusätzlich bestand im Frühling 1994 eine ganz kurze Phase, in der der Beitritt zu FI im Rahmen einer einzelnen Mobilisierungskampagne in einer Fernsehzeitschrift (Tv sorrisi e canzoni) möglich war. Die geworbenen 5.000 Mitglieder wurden aber ohne feste Institutionalisierung und getrennt von den Clubs fortan von der Parteispitze völlig ignoriert. Erst mit der Verabschiedung des neuen Statuts kam ihnen eine wichtige Funktion zu. Sie konstituierten die Nationale Versammlung Forza Italia und bestätigten am 18. Januar 1997 die Änderungen des Statuts und machten damit den Weg für die allgemeine Öffnung von FI frei. Vgl. Emanuela Poli, a.a.O., S. 47, S. 120 .

73 Vgl. ebenda, S. 48.

74 So wurde die Zusammensetzung des „comitato di presidenza“ verändert, das aber nur sehr selten und unregelmäßig tagte. Berlusconi schuf den Posten eines „coordinatore nazionale“, der allerdings nicht im Statut vorgesehen war, aber durch die Nähe zu Berlusconi praktisch legitimiert wurde. Mit einigen Personen der Fininvest bildete Berlusconi einen inoffiziellen Führungszirkel, der ebenfalls nicht im Statut vorkam. Vgl. ebenda, S. $81-86$. 
präsidentielle Prinzip erhalten werden - für Poli ein Konflikt zwischen zwei Grundsätzen: der inneren Demokratie und dem Präsidentialismus Berlusconis. ${ }^{75}$ Es wurde schließlich ein neues Statut ausgearbeitet und verabschiedet, das eine grundlegende Veränderung mit sich brachte: die Öffnung von Forza Italia für eine Massenmitgliedschaft.

Grasmück weist darauf hin, dass FI hiermit eine verspätete Normalisierung erfahren hat. ${ }^{76}$ Bis zum Jahresende 1997 ließen sich 140.000 Mitglieder registrieren; im Jahr 2000 erreichte die Entwicklung mit 312.000 Mitgliedern ihren Höhepunkt, sank aber bis 2002 wieder um 90.000 ab. ${ }^{77}$ Anders als die Mitglieder der Clubs Forza Italia verfügt die neue Mitgliedschaft über einige Mitspracherechte innerhalb der Gesamtorganisation. Auf lokaler Ebene kann sie fortan über Delegierte die Vorsitzenden in den Städten und Provinzen wählen. Die Vorsitzenden der Regionen werden allerdings nach wie vor vom Präsidenten von Forza Italia ernannt. Schließlich wählen die Mitglieder auch Delegierte für den „congresso nazionale“, der im April 1998 erstmals mit 1704 gewählten und 1373 assoziierten Delegierten stattfand. Der „congresso nazionale“ ist als das offiziell höchste Organ der Partei zuständig für die Vorgabe ihrer politischen Richtung, für Veränderungen am Statut und die Wahl des Präsidenten und soll mindestens alle drei Jahre zusammenkommen. In der Zwischenzeit werden die Kompetenzen vom „consiglio nazionale“ wahrgenommen. Entscheidend ist aber das „comitato di presidenza“, das zum Beispiel über die Zusammensetzung der Kandidatenlisten bei Wahlen entscheidet. ${ }^{78}$

Bezüglich der innerparteilichen Demokratie ist aber festzuhalten, dass erstens die Einflussrechte der Mitgliedschaft nicht die Ebene der Regionen betreffen. Zweitens wählen die Mitglieder zwar Delegierte in den „consiglio nazionale“ und das „comitato di presidenza“, doch ist die Wahl eine Farce. Während für Berlusconi keine Vorgaben bestehen, kann die Basis in den „consiglio nazionale“ nur 50 Mitglieder entsenden. Das gleiche gilt für das „,omitato di presidenza“. Die Mitglieder wählen sechs Delegierte, während Berlusconi so viele benennen kann, wie er will. ${ }^{79}$ Nicht nur die Zusammensetzung, sondern auch die Beliebigkeit der Arbeitsweise und Zusammenkunft untermauert die Machtstellung des Präsidenten. Daher resümiert Grasmück im Einklang mit seiner Theorie der charismatischen Partei, dass Berlusconi als charismatische Führungsgestalt nahezu uneingeschränkt herrscht und die Elemente innerparteilicher Demokratie durch ein rigides Kontrollsystem von oben massiv konterkariert werden. ${ }^{80}$ Dem ist eher zuzustimmen als der Feststellung von Poli, dass aus der

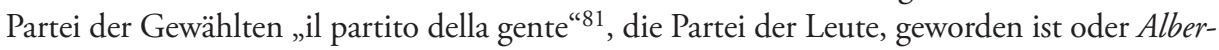
tis Bezeichnung als Mitgliederpartei. Die Mitglieder sind durch eine Zunahme von Partizipationsmöglichkeiten innerhalb der Partei zwar wichtiger geworden und näher an die Parteielite heran gerückt. Diese innerparteiliche Demokratie ist allerdings noch rudimentär, was die Etablierung parteieigener Unterorganisationen auch nicht geändert hat. ${ }^{82}$

75 Ebenda, S. 116.

76 Damian Grasmück, a.a.O., S. 387.

77 Paolo Alberti, a.a.O., S. 246.

78 Emanuela Poli, a.a.O., S. 123 - 130.

79 Im März 2001 bestand dieses Komitee aus nicht weniger als 58 Mitgliedern, von denen nur sechs nicht von Berlusconi ernannt wurden. Vgl. Damian Grasmück, a.a.O., S. 414.

80 Ebenda, S. 389.

81 Emanuela Poli, a.a.O., S. 125.

82 So gehört zu Forza Italia seit 1997 eine eigene Jungend- („Giovani per la libertà“) und eine eigene Rentnerorganisation („Forza Italia - Seniores“). Diese Parteiorganisationen können als ein Teil 
Auch bei der Parteielite und ihren Handlungsweisen ergeben sich nach der Strukturreform 1997 wesentliche Veränderungen. Erstens versucht FI entgegen dem Trend anderer europäischer Großparteien, dem Prinzip der schlanken Organisation treu zu bleiben. Trotz der Schaffung verschiedener Büros und Abteilungen ist die Anzahl der hauptamtlichen Funktionäre innerhalb der Parteizentrale nie über 55 gestiegen. Viele Aufgaben in den Büros, die in traditionellen Parteien Funktionäre übernehmen, werden bei FI von Parlamentariern erledigt. ${ }^{83}$ Ein Heer von Mitarbeiterstäben und Assistenten in der Parteizentrale lässt sich bei FI nicht finden. Zweitens hat sich die Einnahmenstruktur von FI zu einem Drei-Säulen-Modell verändert. So stammten 30,5 Prozent der Einnahmen im Jahr 2000 aus Mitgliedsbeiträgen, während die staatlichen Zuschüsse 59,7 Prozent und Spenden 9,4 Prozent ausmachten. Trotz der niedrigen Personalkosten ${ }^{84}$ ist dieses Geld dringend nötig, da Forza Italia stattdessen in den Bereichen Kommunikation, Marketing, Demoskopie und Personalrekrutierung (für die unteren Ebenen der Parteiorganisation) ein intensives „outsourcing“ der Parteiarbeit betreibt. ${ }^{85}$ In jedem Wahlkampf wurde bislang auf die Umfrageinstitute Datamedia und Diakron zurückgegriffen, die die Kommunikationsstrategie sowie Koalitionen und Programme vorbereiteten. So wurden die Regionalwahl-Kandidaten 1999 von deren Human-Resource-Management-Experten ausgesucht. ${ }^{86}$ War Forza Italia vorher mit den Kommunikations- und Marketingfirmen der Fininvest verschmolzen, haben sich diese nach der Reform 1997 abgetrennt und sind fortan externe Firmen, obwohl sie noch immer die Parteiarbeit organisieren. Dabei unterliegen sie aber mehr der neuen Organisationslogik von FI, das heißt die gefundenen Kandidaten werden von Parteigremien abgesegnet.

Erhalten haben sich drittens Berlusconis öffentliche Absagen an das Berufspolitikertum. Nach Alberti verkörpert Forza Italia mit dieser Position eine neue Art der Legitimation politischer Parteien. ${ }^{87}$ Die Gegenüberstellung von einfachem Volk und abgehobener Elite ist aber in populistischen Parteien ein verbreitetes Phänomen und als Legitimation einer Partei nicht neu. ${ }^{88}$ Zudem zeigt Alberti selbst, dass der Anteil der wiedergewählten Abgeordneten von FI zwischen 1996 und 2001 schon bei der Hälfte lag. Somit kann dieses Legitimationsmodell seine Gültigkeit höchstens für die Anfangsphase beanspruchen. ${ }^{89}$

der typischen Aufstiegskanäle zur politischen Führungsebene verstanden werden, mit denen zunehmend der Trend zur Professionalisierung der Politik und damit der politischen Elite zum Ausdruck kommt.

83 Ebenda, S. 199.

84 Im Jahr 2000 lag der Anteil der Personalkosten an der Menge der Gesamtausgaben bei nur 3,6 Prozent, nachdem er 1996 mit 15,5 Prozent seinen Höchststand erreicht hatte und von dort an kontinuierlich sank. Vgl. Paolo Alberti, a.a.O., S. 255.

85 Während sich Forza Italia zu Beginn ausschließlich aus staatlichen Zuschüssen finanzierte, kommt seit der Organisationsreform 1997 knapp ein Drittel der Finanzmittel aus den Beiträgen der Mitglieder. Vgl. Emanuela Poli, a.a.O., S. 275.

86 Diese professionellen Headhunter wählten Personen mit dem größtmöglichen Wählerpotential aus, die dann nach der Zustimmung von Berlusconi der „conferenza dei coordinatori regionali“ zur Bestätigung vorgeschlagen wurden. Vgl. ebenda, S. 147.

87 Paolo Alberti, a.a.O.

88 Frank Decker, Der neue Rechtspopulismus, Opladen 2004, S. 34.

89 Dass Berlusconi medienwirksam die Berufspolitiker als Diebe bezeichnet, ist der Versuch, das Bild des sauberen Außenseiters aufrecht zu erhalten, was im Widerspruch zu seiner jahrelangen Rolle als Regierungschef, aber im Einklang mit populistischen Grundzügen steht. Vgl. Ugo Magri, Dai politici di professione chiacchiere e soldi rubati, in: La Stampa vom 20. Februar 2004, S. 5. 
Der letzte, vierte, Aspekt betrifft die Wählerschaft als Verbindungselement zur Gesamtgesellschaft. Alessandro Chiaramonte zeigt zwar, dass die Volatilität bei der Parlamentswahl (für die Abgeordnetenkammer) 2001 mit 21,9 geringer war als 1994 mit 36,7 Prozent. Dennoch erwartet er in Zukunft aufgrund einer wachsenden „fedeltà leggera“ (Volatilität) nicht unbedingt eine Stabilisierung auf einem niedrigeren Niveau. ${ }^{90}$ Bei der letzten Wahl 2006 hat sich diese Erwartung nicht bestätigt. Die vergleichsweise niedrige Volatilität von etwa neun Prozent wertet Stefan Köppl als eine Konsolidierung des bipolaren Parteiensystems. $^{91}$

Abbildung 3 verdeutlicht die intra-organisatorische Entwicklung von Forza Italia im Übergang von der Gründungs- zur Konsolidierungsphase. Hervorzuheben ist, dass sich die Extrempositionen im Verhältnis der Teilelemente zueinander nach der Organisationsreform 1997 relativiert haben.

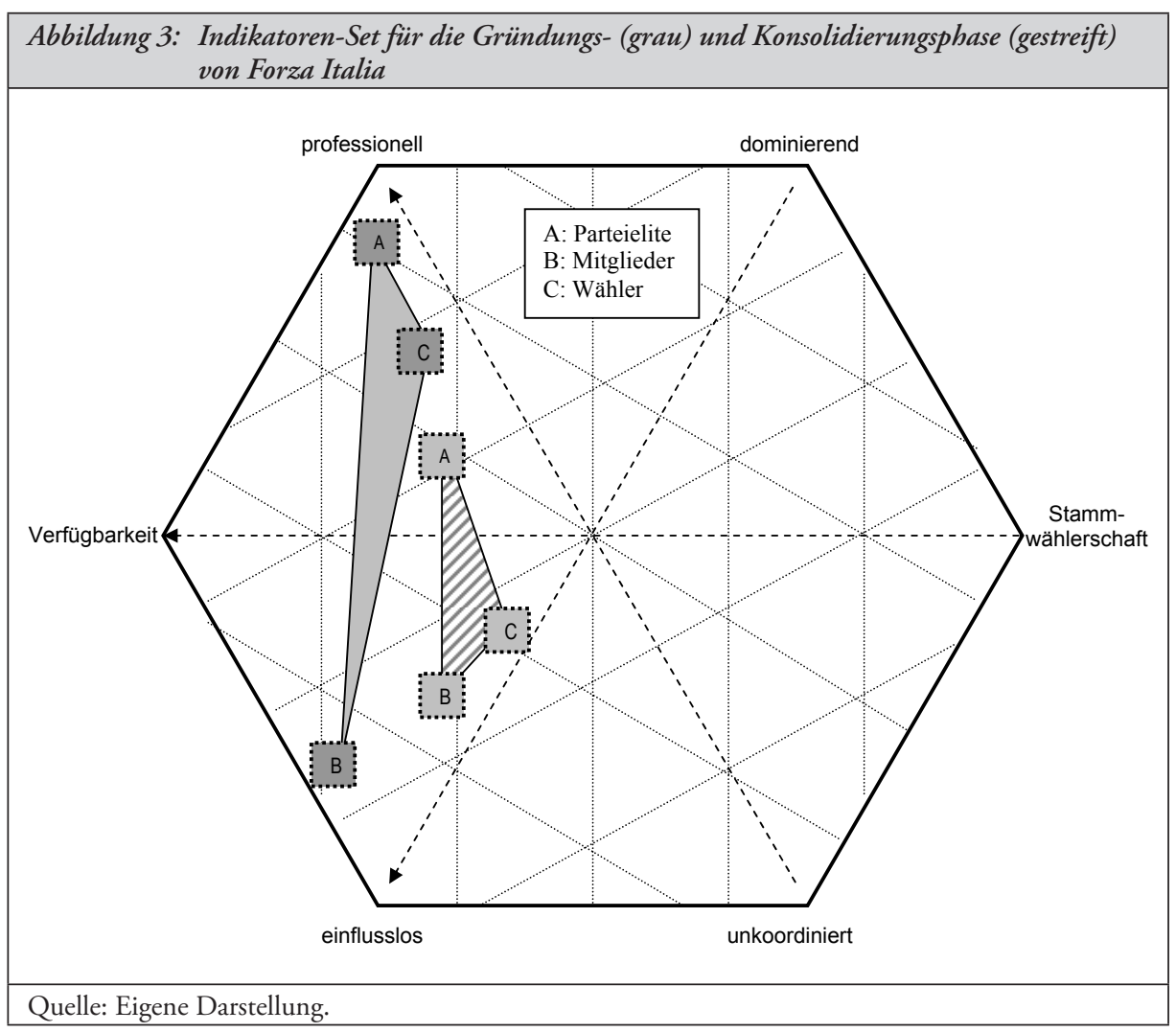

90 Alessandro Chiaramonte, a.a.O., S. 185.

91 Vgl. Stefan Köppl, Die italienischen Parlamentswahlen 2006, a.a.O., S. 760 f. 


\section{Bewertung von Forza Italia aus stadientypologischer Perspektive}

Besonders die beschränkten Mitspracherechte der Mitgliedschaft machen deutlich, dass Forza Italia nicht dem Typus der Kartell-Partei und noch viel weniger der Volkspartei entspricht (vgl. Abbildung 4). Schmid und Zolleis weisen darauf hin, dass selbst unter den Prämissen der vierten Phase des Parteienwandels die Mitgliedschaft aufgrund ihrer VetoPosition innerhalb der Parteiorganisation eine für die Parteiführung zu beachtende Instanz ist $^{92}$, während nach Katz und Mair die Mitgliedschaft der Kartell-Partei unter Umständen sogar noch mehr Rechte hat als die der Volkspartei (nur atomisierter ist). Demnach hat sich Forza Italia nicht zur Mitgliederpartei gewandelt, sondern nur seine Extremposition verlassen und sich dieser angenähert, ist von ihr aber noch weit entfernt. Der Unterschied ist, dass die Mitglieder von Forza Italia innerhalb der Partei keine größere Rolle spielen können, während die idealtypischen Mitglieder der Kartell-Partei dies nicht mehr unbedingt wollen. Zahlenmäßig erscheint FI vielleicht wie eine Mitgliederpartei - von der Qualität der Mitgliedschaft ist sie es aber nicht. Daher unterscheidet sie sich auch im Verhältnis zu den anderen Teileinheiten. Zwischen der Mitgliedschaft und der Parteielite ist die Distanz bei FI zwar durch die Reform 1997 kleiner geworden, ist aber immer noch größer als bei der Kartell-Partei und vor allem der Volkspartei. Genau umgekehrt ist es im Verhältnis von Mitgliedschaft und Wählerschaft. Der Abstand in der Bedeutung zwischen diesen Teileinheiten ist bei FI geringer als bei der Kartell-Partei und erheblich geringer als bei der Volkspartei.

Auch hinsichtlich der Wählerschaft von Forza Italia nach 1997 wird der große Unterschied zur Volkspartei deutlich, während hier mit der Kartell-Partei in etwa Übereinstimmung besteht. War die gestiegene Verfügbarkeit zu Beginn der 1990er Jahre noch die Prämisse für die Gründung von Forza Italia, hat sich dieser Zustand bei den letzten beiden Wahlen wieder relativiert. Dennoch kennzeichnet die Abschwächung der traditionellen Wählermilieus im Norden und in der Mitte Italiens, die Entideologisierung der Gesellschaft und die erhöhte Bereitschaft zur politischen Umorientierung diese Phase. Somit kann die These von Paolo Alberti schon allein aufgrund der gesellschaftlichen Veränderungen Italiens nicht zutreffen, denn die italienische Gesellschaft am Ende des 20. Jahrhunderts ist nicht mehr jene aus der Hochphase der Volksparteien der 1960er Jahre (vorsichtig eingeordnet). Die Parteielite von Forza Italia stellt sich darauf ein, indem sie den Wählern mit einem entideologisierten und pragmatischen Programm gegenübertritt. Durch die minimale Orientierung an der rudimentären Mitgliedschaft ist die Distanz zwischen der Parteielite und der Wählerschaft größer geworden. Damit entspricht sie allerdings in etwa jener der Kartell-Partei.

Die Parteielite von Forza Italia befindet sich hinsichtlich ihrer Professionalisierung zwischen der Kartell-Partei und der Volkspartei, da jeweils zwei Faktoren einem Typus entsprechen (vgl. Abbildung 4). FI verfügt erstens nicht über die großen parteieigenen Beraterstäbe, sondern verfolgt das Prinzip einer schlanken Organisation und unterscheidet sich mit ihrem schmalen Parteiapparat von der Tendenz der professionalisierten Wählerpartei und der Kartell-Partei, eine breite „professionelle Funktionärselite “93 aufzubauen. Berufspolitikertum und der schmale Parteiapparat sind vielmehr Faktoren, die sich in den Parametern 


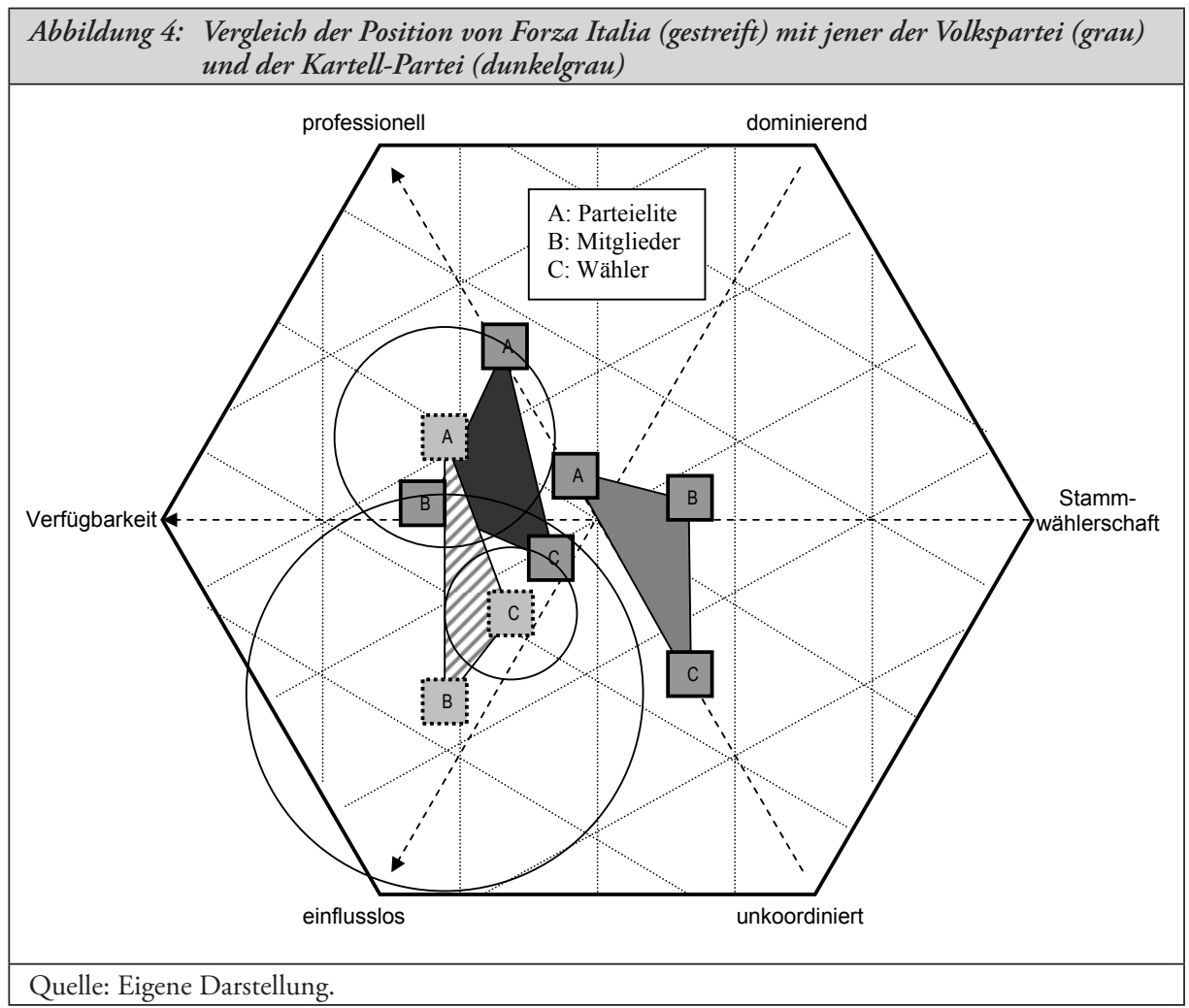

der Volkspartei bewegen. Dadurch ist FI allerdings nicht minder professionell. Die konsequente Auslagerung der Wahlkampfführung und allgemeiner Kampagnenarbeit an externen Sachverstand (aus Berlusconis Firmenimperium) ist bei FI noch intensiver als bei der Kartell-Partei. So schreibt Poli, dass der Rückgriff auf externe Berater und Experten aus den Bereichen Kommunikation, Marketing und Human-Resource-Management durchaus bei vielen europäischen und amerikanischen Parteien Standard ist - allerdings nicht in der Intensität wie bei Forza Italia. ${ }^{94}$ Im Einklang mit der Kartell-Parteien-These steht außerdem die Art der Finanzierung von FI: Im Jahr 2000 kamen bei Forza Italia, wie oben ausgeführt, 57,7 Prozent aller Einnahmen aus staatlichen Zuschüssen, aber nur 30,5 Prozent aus Mitgliedsbeiträgen. ${ }^{95}$ In der Summe charakterisieren also zwei Faktoren die Parteielite von FI als Volkspartei, zwei dagegen als Kartell-Partei.

94 Vgl. Emanuela Poli, a.a.O., S. 201.

95 Zum Vergleich: Bei der SPD machten im Jahr 2002 die Mitgliederbeiträge einen Anteil von 49,2 Prozent aus, während die staatlichen Zuschüsse einem Anteil von 30,9 Prozent entsprachen. Vgl. Ulrich von Alemann / Thelse Godewerth, Die Parteiorganisation der SPD. Erfolgreiches Scheitern, in: Josef Schmid / Udo Zolleis (Hrsg.), a.a.O., S. 158 - 171, S. 169. 


\section{Forza Italia auf dem Weg zur Kartell-Partei}

Jede Partei hat ihre eigene Geschichte, die ihr ein gewisses Maß an Individualität verleiht. Trotz aller Unterschiede hat die Forschung zum Parteienwandel typologische Gemeinsamkeiten ausgemacht, die die Zuordnung einzelner Realtypen zu verschiedenen Entwicklungsstadien erlauben. Die Veränderungen der Parteien gehen kontinuierlich weiter, so dass viele Anzeichen für eine vierte Phase sprechen. Letztendlich lässt sich aber auch mit dieser Untersuchung das Modell der Kartell-Partei weder widerlegen noch endgültig beweisen. Wenn aber die Eigenschaften der Kartell-Partei von Katz und Mair als derzeitiger Durchschnitt des parteientypologischen Entwicklungstandes gelten, vollzieht FI eine Entwicklung weg von extremen Positionen hin zu diesem Durchschnitt im Sinne einer zunehmenden Mäßigung seiner Organisationsformen. Dabei gleichen zwei ihrer intra-organisatorischen Konstellationen jenen der Kartell-Partei: die Position der Wählerschaft und deren Distanz zur Parteielite. Auf der anderen Seite stellen drei von sechs Vergleichspunkten (vgl. Abbildung 4) aber immer noch Extrempositionen dar, die nicht nur außerhalb der Parameter der Volkspartei, sondern auch der Kartell-Partei liegen. Die Rolle der Mitgliedschaft und ihr Verhältnis zu den anderen Teilelementen gehen wesentlich über den Stand der Kartell-Partei hinaus. Auch wenn die Parteielite als letzter Punkt noch Ähnlichkeiten mit dem Typus der Volkspartei aufweist, handelt es sich bei Forza Italia wahrlich nicht um eine solche.

Angesichts der fließenden Grenzen der Stadientypologie erweist sich die Kategorie einer „catch-all-Partei hoch drei“ als unsinnig, da sie der Diskrepanz zwischen Realtypen und Idealtypen nicht Rechnung trägt. Es ist festzuhalten, dass der Einzelvergleich von Forza Italia mit der Stadientypologie, wie nicht anders erwartet, Unterschiede zu den Idealtypen hervorgebracht hat; dennoch entsprechen die intra-organisatorischen Strukturen von Forza Italia nach der Reform von 1997 viel mehr den Dimensionen der Kartell-Partei als jenen der Volkspartei. Zudem hat sich die Stadientypologie als robust genug erwiesen, auch einen Sonderfall wie FI detailliert zu analysieren. Es bestand in diesem Fall also kein Theorie-, sondern ein Anwendungsdefizit. 